\title{
Transient temperature distribution and thermal stresses in a thick plate subjected to random pulses
}

\author{
Sonal Bhoyar ${ }^{1 *}$, Vinod Varghese ${ }^{2}$, Lalsingh Khalsa ${ }^{1}$ \\ ${ }^{1}$ Department of Mathematics, M.G. College, Armori, Gadchiroli, India \\ ${ }^{2}$ Department of Mathematics, Smt. Sushilabai Bharti Science College, Arni, Yavatmal, India \\ E-mail: sonalwbhoyar@gmail.com
}

Received 20 August 2019, Revised 4 October 2019, Accepted 21 October 2019

\begin{abstract}
This paper deals with the determination of second-order statistics (the mean of each component and correlations between components) of the quasi-static temperature distribution and thermal stresses on a thick plate under thermal load. The heated thick circular plate with random internal heat generation acting at random instants of time, in addition to sectional heat supply in the form of random impulses on the upper face, while the lower face is kept at zero temperature, and the fixed circular edge thermally insulated in considered for investigation. A type of Laplace transform method is employed to obtain the analytical solutions for the temperature and stresses statistics. The effects of the statistics of the random temperature distribution and its associated stresses are signified graphically that highlight the developments proposed in this paper.
\end{abstract}

\section{Keywords: Random pulse; temperature; thermal stresses; moments; correlation.}

\section{Introduction}

Usually forecasting the mechanical or thermal loads acting on structural elements is incredibly troublesome. There's no doubt that their material properties are random variables and so are sometimes explicit in terms of average values with hooked up uncertainties. Thus, the consideration of safety was familiarized into the operational design. The present design methods based on the safety parameters cannot quantitatively estimate the safety of structures. To sidestep such a problem, the probability theory and mathematical statistics have been applied to many engineering problems. At this time, the application of probabilistic methods to engineering problems has been broadened to the segment of heat transfer. As structures subjected to extreme thermal load currently hold a prominent position in industries, the heat conduction analysis and associated thermal stresses in solids have drawn attention.

In studies on the thermoelastic responses of the bodies subjected to random pulse, solely stationary random processes have been targeted so far. Thus, when time functions included as a random internal heat supply or/and the thermal boundary conditions are equipped with sectional random heat supply, then the mathematical complicity further increases. Therefore, the analyses treated in the framework of the theory of a stationary random process ought to be extended to nonstationary random processes was explained in the highly cited review paper [1]. A short history of the research work associated with random pulse and application of probabilistic methods are discussed in detail as a prerequisite. Mathews and Srinivasan [2] studied physical processes which can be represented by symbolic differential equations involving random functions. Parkus [3] studied random thermal stresses in which he successfully analyzed the thermoelastic problem of a semi-infinite body using probabilistic methods. Samuels [4] conducted a study on heat conduction analysis using probabilistic methods. He analyzed heat conduction in a plate and sphere with randomly fluctuating surface temperature and spatiotemporally random internal heat generation. Srinivasan et al. [5] discussed the unsteady motion of a viscous fluid subjected to random pulses. Hung [6] presented the temperature distribution of straight and circular fins with stochastic root temperature. He obtained the response autocorrelation functions and power spectral densities of temperatures with stationary Gaussian excitation temperatures. Heller [7] developed statistical relations for the temperature response in an infinitely thick slab subjected to randomly varying surface temperatures. Lenyuk et al. [8,9] investigated the case of an elastic halfspace by taking random boundary conditions and an isotropic homogeneous symmetric body by taking the external medium temperature to be an arbitrary function of time and determined the stresses. Becus [10] bestowed a solution to the heat conduction problem with random heat supply and random boundary conditions. Vasseur and Singh [11] analytically obtained relationships between the autocorrelation functions of heat generation and heat flow within the three-dimensional steady heat conduction of a solid body. Nielsen [12] proposed the work to deal with the case of heat conduction with random heat generation and obtained cross-correlation between them. Val'kovskaya and Lenyuk [13] analyzed the stochastic temperature field in a two-layer solid disk subjected to heat sources for which power is a random function of time and radial coordinates. Recently, Sugano et al. [14] used probability theory to analyze the heat conduction and thermal stress problems of functionally graded plates with randomly varying surface temperature assumed to be a homogeneous random field. 
Chiba and Sugano [15-19] analyzed the thermoelastic problem of homogeneous bodies, functionally graded plates and nonhomogeneous plates with a random thermal conductivity and coefficient of thermal expansion using various boundary conditions. They have not however considered any thermoelastic problem subjected to random temperature distribution, which satisfies the time-dependent heat conduction equation. From the previous literature regarding thick plate, as well-thought-out, it was observed by the author that no analytical procedure has been reported, considering thick clamped plate subjected to random heat inputs on its upper face. Thus, our study highlights the behaviour of thermal stresses in a twodimensional thick elastic body with random internal heat sources and subjected to an additional random sectional heat supply impacted on the upper layer. The success of this novel analysis lays with the mathematical methods with a way more straightforward approach for improvement for the design in terms of material usage and performance in engineering drawbacks, significantly within the determination of thermoelastic behaviour in thick plate. Thus, motivated by applications to physical and biological problems in thick structural profile subjected to random temperature, the author considered the probabilistic aspects of the thermal problem for this paper. During the design and building of nuclear reactors and the fabrication of foundation of furnaces, pressure vessels, et cetera, subjected to random temperature are often attributed to thermal stresses.

\section{Formulation of the Problem}

Let us consider a thick circular plate with a radius $a$ and constant thickness $\ell$, with random internal heat generation acting at random instants of time, in addition to sectional heat supply in the form of random impulses on the upper face, while the lower face was kept at zero temperature, and the fixed circular edge thermally insulated in considered for investigation. The plate material is considered to be isotropic, and the material properties are assumed to be constant, as shown in figure 1 .

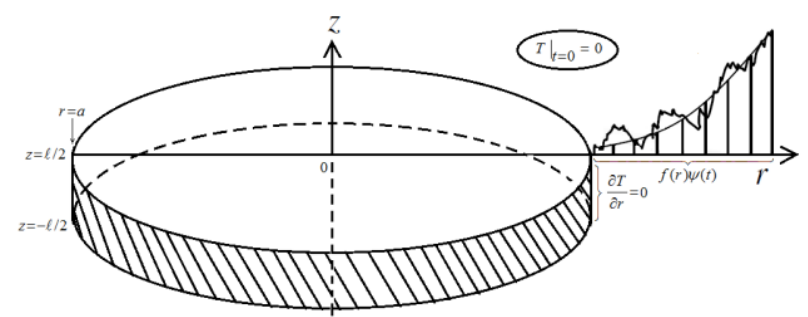

Figure 1. The physical configuration of a thick plate.

\subsection{Temperature Distribution}

We assume that the plate is subjected to pulses of random strength acting at random instants of time. At the time $t=0$, a temperature gradient which is a transient function is set-up, the system being initially at rest. Thus, we assume that the temperature distribution $T=T(r, z, t)$ in terms of the stochastic integral as

$$
\begin{aligned}
T & =\sum_{m=1}^{\infty} \sum_{n=1}^{\infty} A_{n} J_{0}\left(\alpha_{n} r\right) \sinh \left[\gamma_{m}\left(z+\frac{\ell}{2}\right)\right] \\
& \times\left(\int_{0}^{t} \exp [-\omega(t-\tau)] \mathrm{dN} \tau+\int_{0}^{t} F(\tau) \exp [-\omega(t-\tau)] d \tau\right)
\end{aligned}
$$

in which $N(\tau)$ presents the number of pulses in time $\tau$, $d N(\tau)$ is the number of pulses in the interval $(\tau, \tau+d \tau)$ that can be represented as $d N(\tau)=\exp (-y) y^{\tau} / \tau ! d \tau$, with $y(y>0)$ is a parameter of distribution, $\tau(\tau=0,1,2, \ldots)$ is a random variable. Let $\alpha_{1}, \alpha_{2}, \ldots$ be the roots of the transcendental equation $J_{1}(\alpha a)=0$, Bessel functions $J_{0}$ and $J_{1}$ is of the first kind of order zero and one, $\omega$ being a constant, $\gamma_{m}$ will be determined later, and the constant $A_{2 n}$ can be found from the nature of the temperature prescribed on the upper face, respectively.

The heat conduction equation for plate [20] is

$\frac{\partial^{2} T}{\partial r^{2}}+\frac{1}{r} \frac{\partial T}{\partial r}+\frac{\partial^{2} T}{\partial z^{2}}+F(t)=\frac{1}{\kappa} \frac{\partial T}{\partial t}$

where the thermal diffusivity of the material is $\kappa=k / \rho C_{v}$, with $k$ as the thermal conductivity, $\rho$ is the density and $C_{v}$ is the calorific capacity, respectively.

The boundary conditions for temperature are

$\left.T\right|_{t=0}=\left.\frac{\partial T}{\partial r}\right|_{r=a}=\left.T\right|_{z=-\ell / 2}=0,\left.T\right|_{z=+\ell / 2}=f(r) \psi(t)$

Substituting Eq. (1) into Eq. (2), one obtains

$\gamma_{m}^{2}=\alpha^{2}+\omega / \kappa$

We use the Bessel-Dini formula which has the following form

$$
\begin{aligned}
f(r)= & \left(\frac{\eta}{a}\right)^{2}+\frac{2}{a^{2}} \int_{0}^{a} r f(r) d r+\sum_{n=1}^{\infty}\left(\frac{2}{a^{2}\left[J_{0}\left(\alpha_{n} a\right)\right]^{2}}\right. \\
& \left.\times \int_{0}^{a} f(r) r J_{o}\left(\alpha_{n} r\right) d r\right) J_{o}\left(\alpha_{n} r\right), r \in[0, a)
\end{aligned}
$$

Since the upper surface is subjected to random temperature distribution in a concentric circular region, we assume

$$
f(r)= \begin{cases}1 ; & 0<r<r_{0} \\ 0 ; & r>r_{0}\end{cases}
$$

Hence, we get

$$
f(r)=\sum_{n=1}^{\infty}\left(\frac{2 r_{0} J_{1}\left(\alpha_{n} r_{0}\right)}{a^{2} \alpha_{n}\left[J_{0}\left(\alpha_{n} a\right)\right]^{2}}\right) J_{0}\left(\alpha_{n} r\right)
$$

From Eq. (1), Eq. (3) and Eq. (7), one obtains

$$
A_{n}=\frac{2 \eta_{0} J_{1}\left(\alpha_{n} \eta\right) \psi(t)}{\left.\left\{\begin{array}{l}
a^{2} \alpha_{n}\left[J_{0}\left(\alpha_{n} a\right)\right]^{2} \sinh \left(\gamma_{m} \ell\right) \times \\
\left.\int_{0}^{t} \exp [-\omega(t-\tau)] d N \tau+\int_{0}^{t} F(\tau) \exp [-\omega(t-\tau)] d \tau\right)
\end{array}\right)\right\}}
$$

The temperature change $\tau^{\prime}$ from the initial temperature $T_{i}$ can be obtained as

$$
\begin{aligned}
\tau^{\prime} & =T-T_{i} \\
& =\sum_{m=1}^{\infty} \sum_{n=1}^{\infty} A_{n} J_{0}\left(\alpha_{n} r\right) \sinh \left[\gamma_{m}\left(z+\frac{\ell}{2}\right)\right] \\
& \times\left(\int_{0}^{t} \exp [-\omega(t-\tau)] \mathrm{dN} \tau+\int_{0}^{t} F(\tau) \exp [-\omega(t-\tau)] d \tau\right)
\end{aligned}
$$




\subsection{Thermoelastic Formulation}

The components of strain [20] for an axisymmetric deformation is taken as

$$
\varepsilon_{r r}=\frac{\partial u_{r}}{\partial r}, \varepsilon_{\theta \theta}=\frac{u_{r}}{r}, \varepsilon_{z z}=\frac{\partial u_{z}}{\partial z}, \varepsilon_{z r}=\frac{1}{2}\left(\frac{\partial u_{r}}{\partial z}+\frac{\partial u_{z}}{\partial r}\right)
$$

and the constitutive equations or the generalized Hooke's law is taken as

$$
\begin{aligned}
& \varepsilon_{r r}=\frac{1}{2 G}\left(\sigma_{r r}-\frac{v}{1+v} e\right)+\alpha_{T} \tau^{\prime}, \varepsilon_{\theta \theta}=\frac{1}{2 G}\left(\sigma_{\theta \theta}-\frac{v}{1+v} e\right)+\alpha_{T} \tau^{\prime}, \\
& \varepsilon_{z z}=\frac{1}{2 G}\left(\sigma_{z z}-\frac{v}{1+v} e\right)+\alpha_{T} \tau^{\prime}, \varepsilon_{z r}=\frac{\sigma_{z r}}{2 G}
\end{aligned}
$$

in which the dilatation $e=\varepsilon_{r r}+\varepsilon_{\theta \theta}+\varepsilon_{z z}, u_{r}$ and $u_{z}$ is the displacement components in the radial and axial directions, $G$ being the shear modulus, $v$ is the Poisson's ratio and $\alpha_{T}$ as the thermal expansion coefficient, respectively. Thus, Eq. (11) can be re-written as $\sigma_{r r}=2 \mu \varepsilon_{r r}+\lambda e-\beta \tau^{\prime}, \sigma_{\theta \theta}=2 \mu \varepsilon_{\theta \theta}+\lambda e-\beta \tau^{\prime}$,

$\sigma_{z z}=2 \mu \varepsilon_{z z}+\lambda e-\beta \tau^{\prime}, \sigma_{z r}=2 \mu \varepsilon_{z r}$

in which $\mu=E /[2(1+v)]=G, \quad \lambda=2 v G /(1-2 v)$, $\beta=\alpha(3 \lambda+2 \mu)$ and $(\lambda, \mu)$ are the Lame's constant, respectively. Furthermore, the equilibrium equations in the radial and axial directions disregarding the body forces (i.e. $F_{r}=F_{z}=0$ ) are given by the relationships

$\frac{\partial \sigma_{r r}}{\partial r}+\frac{\partial \sigma_{z r}}{\partial z}+\frac{\sigma_{r r}-\sigma_{\theta \theta}}{r}=0$,

$\frac{\partial \sigma_{r z}}{\partial r}+\frac{\partial \sigma_{z z}}{\partial z}+\frac{\sigma_{r z}}{r}=0$

Substituting Eqs. (10) and (12) into Eq. (13), one obtains Navier's equations for axisymmetric thermoelastic equation as

$$
\begin{aligned}
& \nabla^{2} u_{r}-\frac{u_{r}}{r^{2}}+\frac{1}{1-2 v} \frac{\partial e}{\partial r}-2 \frac{1+v}{1-2 v} \alpha_{T} \frac{\partial \tau^{\prime}}{\partial r}=0 \\
& \nabla^{2} u_{z}+\frac{1}{1-2 v} \frac{\partial e}{\partial z}-2 \frac{1+v}{1-2 v} \alpha_{T} \frac{\partial \tau^{\prime}}{\partial z}=0
\end{aligned}
$$

in which $\nabla^{2}$ in axisymmetrical case denotes

$$
\nabla^{2}=\frac{\partial^{2}}{\partial r^{2}}+\frac{1}{r} \frac{\partial}{\partial r}+\frac{\partial^{2}}{\partial z^{2}}
$$

The displacement function is represented by the Goodier's thermoelastic displacement potential and Love's function [20] as

$$
\begin{aligned}
& u_{r}=\frac{\partial \phi}{\partial r}-\frac{\partial^{2} L}{\partial r \partial z} \\
& u_{z}=\frac{\partial \phi}{\partial r}+2(1-v) \nabla^{2} L-\frac{\partial^{2} L}{\partial^{2} z}
\end{aligned}
$$

in which Goodier's thermoelastic displacement potential $\phi(r, z, t)$ and Love's function $L(r, z, t)$ must satisfy the following governing equations

$$
\begin{aligned}
& \nabla^{2} \phi=\frac{1+v}{1-v} \alpha_{T} \tau^{\prime} \\
& \nabla^{2} \nabla^{2} L=0
\end{aligned}
$$

The stress components are represented by the use of the $\phi(r, z, t)$ and $L(r, z, t)$ as

$$
\begin{aligned}
& \sigma_{r r}=2 G\left\{\left(\frac{\partial^{2} \phi}{\partial r^{2}}-\nabla^{2} \phi\right)+\frac{\partial}{\partial z}\left[v \nabla^{2} L-\frac{\partial^{2} L}{\partial r^{2}}\right]\right\}, \\
& \sigma_{\theta \theta}=2 G\left\{\left(\frac{1}{r} \frac{\partial \phi}{\partial r}-\nabla^{2} \phi\right)+\frac{\partial}{\partial z}\left[v \nabla^{2} L-\frac{1}{r} \frac{\partial L}{\partial r}\right]\right\},
\end{aligned}
$$

$$
\sigma_{z z}=2 G\left\{\left(\frac{\partial^{2} \phi}{\partial z^{2}}-\nabla^{2} \phi\right)+\frac{\partial}{\partial z}\left[(2-v) \nabla^{2} L-\frac{\partial^{2} L}{\partial z^{2}}\right]\right\},
$$$$
\sigma_{r z}=2 G\left\{\frac{\partial^{2} \phi}{\partial r \partial z}+\frac{\partial}{\partial r}\left[(1-v) \nabla^{2} L-\frac{\partial^{2} L}{\partial z^{2}}\right]\right\}
$$

For traction free surface stress functions

$\sigma_{z z}=\sigma_{r z}=0 \quad$ at $z=-\ell / 2$

$\sigma_{r r}=\sigma_{z z}=\sigma_{r z}=0$ at $z=a$

The other stress components depend only on $r$ and $t$ at $z=\ell / 2$ as a special case. Then, the set of equations (1) to (20) constitutes the mathematical formulation for temperature distribution, displacement and thermal stresses developed within solid at any instant.

\section{The Solution of the Problem}

\subsection{Thermoelastic Analysis}

We assume Goodier's displacement potential $\phi(r, z, t)$ as

$$
\begin{aligned}
\phi & =\sum_{m=1}^{\infty} \sum_{n=1}^{\infty} B_{n} J_{0}\left(\alpha_{n} r\right) \sinh \left[\gamma_{m}(z+\ell / 2)\right] \\
& \times\left\{\int_{0}^{t} \exp (-\omega(t-\tau)) d N \tau+\int_{0}^{t} F(\tau) \exp (-\omega(t-\tau)) d \tau\right\}
\end{aligned}
$$

Similarly, the solution for Love's function $L(r, z, t)$ is assumed as

$$
\begin{aligned}
L & =\sum_{m=1}^{\infty} \sum_{n=1}^{\infty} B_{n} J_{0}\left(\alpha_{n} r\right)\left\{K _ { m 1 } \operatorname { s i n h } \left[\gamma_{m}(z+\ell / 2)\right.\right. \\
& \left.+K_{m 2} \cosh \left[\gamma_{m}(z+\ell / 2)\right]\right\} \\
& \times\left\{\begin{array}{l}
t \\
\left.\int_{0} \exp [-\omega(t-\tau)] d N \tau+\int_{0}^{t} F(\tau) \exp [-\omega(t-\tau)] d \tau\right\}
\end{array}\right.
\end{aligned}
$$

Substitute Eqs. (21) and (22) in Eq. (16), one obtains

$$
\begin{aligned}
& u_{r}=\sum^{\infty} \sum^{\infty} B_{n} \alpha_{n} J_{1}\left(\alpha_{n} r\right)\left\{-\gamma_{m} K_{m 1} \cosh \left[\gamma_{m}(z+\ell / 2)\right]\right. \\
& m=1 n=1 \\
& \left.-\left(1-\gamma_{m} K_{m 2}\right) \sinh \left[\gamma_{m}(z+\ell / 2)\right]\right\} \\
& \times\left\{\int_{0}^{t} \exp (-\omega(t-\tau)) d N \tau+\int_{0}^{t} F(\tau) \exp (-\omega(t-\tau)) d \tau\right\} \\
& u_{z}=\sum_{m=1}^{\infty} \sum_{n=1}^{\infty} B_{n}\left\langle\left[\alpha_{n} J_{1}\left(\alpha_{n} r\right)\right] \sinh \left[\gamma_{m}(z+\ell / 2)\right]\right. \\
& +\left\{2(1-v)\left[\alpha_{n} J_{1}\left(\alpha_{n} r\right) / r+J_{0}\left(\alpha_{n} r\right) \gamma_{m}^{2}\right]-J_{0}\left(\alpha_{n} r\right) \gamma_{m}^{2}\right\} \\
& \times\left\{K_{m 1} \sinh \left[\gamma_{m}(z+\ell / 2)+K_{m 2} \cosh \left[\gamma_{m}(z+\ell / 2)\right]\right\}\right\rangle \\
& \times\left\{\begin{array}{l}
t \\
\left.\int_{0} \exp [-\omega(t-\tau)] d N \tau+\int_{0}^{t} F(\tau) \exp [-\omega(t-\tau)] d \tau\right\}
\end{array}\right.
\end{aligned}
$$

Substituting Eqs. (21) and (22) in Eq. (19), one obtains the stresses as 


$$
\begin{aligned}
& \sigma_{r r}=\sum_{m=1}^{\infty} \sum_{n=1}^{\infty} 2 G B_{n}\left\langle\left[-\alpha_{n} J_{1}\left(\alpha_{n} r\right) / r-\gamma_{m}^{2} J_{0}\left(\alpha_{n} r\right)\right]\right. \\
& \times \sinh \left[\gamma_{m}(z+\ell / 2)\right]+\gamma_{m}\left\{K_{m 1} \cosh \left[\gamma_{m}(z+\ell / 2)\right]\right. \\
& \left.+K_{m 2} \sinh \left[\gamma_{m}(z+\ell / 2)\right]\right\} \\
& \left.\times v\left[\alpha_{n} J_{1}\left(\alpha_{n} r\right) / r+\gamma_{m}^{2}\right]\left[\alpha_{n} J_{1}\left(\alpha_{n} r\right) / r+J_{0}\left(\alpha_{n} r\right)\right]\right\rangle \\
& \times\left\{\int_{0}^{t} \exp (-\omega(t-\tau)) d N \tau+\int_{0}^{t} F(\tau) \exp (-\omega(t-\tau)) d \tau\right\} \\
& \sigma_{\theta \theta}=\sum_{m=1}^{\infty} \sum_{n=1}^{\infty} 2 G B_{n}\left\langle\gamma_{m}^{2} J_{0}\left(\alpha_{n} r\right) \sinh \left[\gamma_{m}(z+\ell / 2)\right]\right. \\
& +\gamma_{m}\left[(v-1) \alpha_{n} J_{1}\left(\alpha_{n} r\right) / r+v \gamma_{m}^{2} J_{0}\left(\alpha_{n} r\right)\right]\left\{K_{m 1}\right. \\
& \left.\times \cosh \left[\gamma_{m}(z+\ell / 2)+K_{m 2} \sinh \left[\gamma_{m}(z+\ell / 2)\right]\right\}\right\rangle \\
& \times\left\{\int_{0}^{t} \exp (-\omega(t-\tau)) d N \tau+\int_{0}^{t} F(\tau) \exp (-\omega(t-\tau)) d \tau\right\} \\
& \sigma_{z z}=\sum_{m=1}^{\infty} \sum_{n=1}^{\infty} 2 G B_{n}\left\langle-\alpha_{n} J_{1}\left(\alpha_{n} r\right) \sinh \left[\gamma_{m}(z+\ell / 2)\right] / r\right. \\
& +\gamma_{m}\left[(2-v) \alpha_{n} J_{1}\left(\alpha_{n} r\right) / r+(1-v) \gamma_{m}^{2} J_{0}\left(\alpha_{n} r\right)\right] \\
& \times\left\{K_{m 1} \cosh \left[\gamma_{m}(z+\ell / 2)+K_{m 2} \sinh \left[\gamma_{m}(z+\ell / 2)\right]\right\}\right\rangle \\
& \times\left\{\int_{0}^{t} \exp (-\omega(t-\tau)) d N \tau+\int_{0}^{t} F(\tau) \exp (-\omega(t-\tau)) d \tau\right\} \\
& \sigma_{r z}=\sum_{m=1}^{\infty} \sum_{n=1}^{\infty} 2 G B_{n} \alpha_{n} \gamma_{m} J_{1}\left(\alpha_{n} r\right)\left\langle\cosh \left[\gamma_{m}(z+\ell / 2)\right]\right. \\
& \begin{array}{l}
m=1 n=1 \\
-v \gamma_{m}\left\{K _ { m 1 } \operatorname { s i n h } \left[\gamma_{m}(z+\ell / 2)\right.\right.
\end{array} \\
& \left.\left.+K_{m 2} \cosh \left[\gamma_{m}(z+\ell / 2)\right]\right\}\right\rangle \\
& \times\left\{\int_{0}^{t} \exp (-\omega(t-\tau)) d N \tau+\int_{0}^{t} F(\tau) \exp (-\omega(t-\tau)) d \tau\right\}
\end{aligned}
$$

Using Eqs. (25)-(28) in Eq. (20), one obtains

$$
K_{m 1}=0, K_{m 2}=-1 / v \gamma_{m}
$$

\subsection{Product Densities}

We assume that the probability of occurrence of one pulse in $d \tau$ is proportional to $d \tau$ while the probability that there occur $n(n>1)$ pulses in $d \tau$ is of a negligible smaller order of magnitude than $d \tau$. If $p(N)$ is the probability that $n$ pulses occur in $d \tau$, then

$$
\begin{aligned}
& p(1)=\psi_{1}(\tau) d \tau, p(n)=o(d \tau)(n>1) \\
& p(0)=1-\sum_{n \geq 1} p(n)=1-\psi_{1}(\tau) d \tau+o(d \tau)
\end{aligned}
$$

The expectation values of the power of the random variable $d N(\tau)$ are taken as follows

$$
\begin{aligned}
& \varepsilon\{d N(\tau)\}=p(1)+o(d \tau)=\psi_{1}(\tau) d \tau+o(d \tau), \\
& \begin{aligned}
\varepsilon\left\{[d N(\tau)]^{n}\right\} & =p(1)+o(d \tau)=\psi_{1}(\tau) d \tau+o(d \tau) \\
& =\varepsilon\{d N(\tau)\}+o(d \tau)
\end{aligned}
\end{aligned}
$$

in which $\psi_{1}(\tau)$ is the product density of degree one and note that the $\psi_{1}(\tau) d \tau$ is probability magnitude it is integral over $\tau$ yields only the mean number of pulses distributed over the range of integration.

Now consider $\psi_{2}\left(\tau_{1}, \tau_{2}\right)$ as a product density of degree two when the intervals do not overlap $\psi_{2}\left(\tau_{1}, \tau_{2}\right) d \tau_{1} d \tau_{2}=\varepsilon\left\{d N\left(\tau_{1}\right) d N\left(\tau_{2}\right)\right\}$

which is also equal to the joint probability that a pulse lies in $d \tau_{1}$ and a pulse lies in $d \tau_{2}$ when $d \tau_{1}, d \tau_{2}$ do not overlap. But when the intervals overlap, a degeneracy occurs, and

$$
\begin{aligned}
\varepsilon\left\{d N\left(\tau_{1}\right) d N\left(\tau_{2}\right)\right\} & =\varepsilon\left\{\left[d N\left(\tau_{1}\right)\right]^{2}\right\} \\
& =\varepsilon\left\{d N\left(\tau_{1}\right)\right\}=\psi_{1}\left(\tau_{1}\right) d \tau_{1}
\end{aligned}
$$

\subsection{Correlation of Temperature}

The product densities are useful in obtaining the moments as well as the correlation of temperature distribution. Let assume that the $F(\tau)$ characterizing the temperature is a random function of $\tau$. The points of the time axis corresponding to the time of occurrence of pulses are expected to be distributed by Poisson's law with a constant average density $\theta$.

The product density is given by

$\psi_{1}(\tau)=\theta, \psi_{2}\left(\tau_{1}, \tau_{2}\right)=\theta^{2}$

On the distribution of $F(\tau)$ the following restriction is imposed taking $\mu>0, \tau_{2}>\tau_{1}$ as

$$
\begin{aligned}
& \varepsilon\{F(\tau)\}=I, \text { a constant } \\
& \varepsilon\left\{F\left(\tau_{1}\right) F\left(\tau_{2}\right)\right\}=\sin \beta \tau_{1} \exp \left[-\mu\left(\tau_{2}-\tau_{1}\right)\right]
\end{aligned}
$$

The expression for $\varepsilon\{T(r, z, \tau)\}$ is given by

$$
\begin{aligned}
& \varepsilon\{T(r, z, t)\}=\sum_{m=1}^{\infty} \sum_{n=1}^{\infty}\left(A_{n} / \omega\right) J_{0}\left(\alpha_{n} r\right) \\
& \quad \times \sinh \left\{\gamma_{m}[z+\ell / 2]\right\}[1-\exp (-t \omega)]\{\theta+I\}
\end{aligned}
$$

Similarly, in case of product density of degree two

$$
\begin{aligned}
\varepsilon\left\{T\left(r, z, t_{1}\right) T\left(r, z, t_{2}\right)\right\}=\sum_{m, m^{\prime} n, n^{\prime}} \sum_{n} J_{0}\left(\alpha_{n} r\right) J_{0}\left(\alpha_{n^{\prime}} r\right) \\
\times \sinh \left\{\gamma_{m}[z+\ell / 2] \sinh \left\{\gamma_{m^{\prime}}[z+\ell / 2]\right\}\right. \\
\times\left\{\frac{e^{-\omega t_{1}-t_{2} \omega^{\prime}}\left(e^{\omega t_{2}}-e^{\omega \tau_{1}}\right)\left(-1+e^{t_{1} \omega^{\prime}}\right) \theta^{2}}{\omega \omega^{\prime}}\right. \\
+ \\
+e^{(\mu+\omega) \tau_{1} \beta \cos \left(\beta \tau_{1}\right)+(\mu+\omega)\left[e^{(\mu+\omega) t_{2}}\right.} \\
\left.\times \sin \left(\beta t_{2}\right)-e^{(\mu+\omega) \tau_{1}} \sin \left(\beta \tau_{1}\right)\right] \\
+\frac{e^{-\omega t_{1}-t_{2} \omega^{\prime}} \theta^{2}\left[e^{\omega t_{1}}\left(-1+e^{t_{1} \omega^{\prime}}\right) \omega-\left(-1+e^{\omega t_{1}}\right) \omega^{\prime}\right]}{\omega \omega^{\prime}\left(\omega+\omega^{\prime}\right)} \\
+\frac{1}{\beta^{2}+(\mu+\omega)^{2}}\left\langle-e^{-\omega t_{1}-\omega^{\prime} t_{2}}\right. \\
\times\left\{\frac{e^{(\mu+\omega) t_{1}}\left[\beta \cos \left(\beta t_{1}\right)-(\mu+\omega) \sin \left(\beta t_{1}\right)\right]}{\mu-\omega^{\prime}}\right. \\
\left.+\frac{\beta\left(\mu+2 \omega+\omega^{\prime}\right)}{\beta^{2}+\omega^{2}+2 \omega \omega^{\prime}+\omega^{\prime 2}}\right\}+e^{-\mu t_{1}-\omega t_{1}+\omega^{\prime}\left(t_{1}-t_{2}\right)}
\end{aligned}
$$




$$
\begin{aligned}
& \times\left\{\frac{e^{(\mu+\omega) t_{1}}\left[\beta \cos \left(\beta t_{1}\right)-(\mu+\omega) \sin \left(\beta t_{1}\right)\right]}{\mu-\omega^{\prime}}\right. \\
& +\frac{e^{(\mu+\omega) t_{1}} \beta \cos \left(\beta t_{1}\right)\left(\mu+2 \omega+\omega^{\prime}\right)}{\beta^{2}+\omega^{2}+2 \omega \omega^{\prime}+\omega^{\prime 2}} \\
& \left.\left.-\frac{e^{(\mu+\omega) t_{1}} \sin \left(\beta t_{1}\right)\left[-\beta^{2}+\omega(\mu+\omega)+(\mu+\omega) \omega^{\prime}\right]}{\beta^{2}+\omega^{2}+2 \omega \omega^{\prime}+\omega^{\prime 2}}\right\}\right) \\
& \left.+\frac{e^{-\omega t_{1}-t_{2} \omega^{\prime}}\left[-1+e^{t_{1}\left(\omega+\omega^{\prime}\right)}\right]}{\omega+\omega^{\prime}}\right\}
\end{aligned}
$$

\subsection{Moments Subjected to Random Pulses}

The required statistics are obtained for the pulse in the interval $(\tau, \tau+d \tau)$. The general expression derived for the moments and correlation of the pulse requires neither independence of intervals between time base points nor independence of the pulses. When the time base is a Poisson point process, and the pulse shapes are independent, stronger results become available and hence we will be able to calculate joint characteristic functions for the pulse process, thus providing a more complete statistical description. The technique used here results in general expressions for the moments and correlation of temperature distribution and thermal stresses of such pulse. Now following [5], in case of product density of degree one, we assume

$$
\varepsilon\{d N(\tau)\}=\bar{C} d \tau
$$

$\varepsilon\{\psi(\tau)\}=D$, a constant.

in which $\bar{C}$ is the constant average density and $\varepsilon$ denotes the correlation function.

Since the probability of occurrence of one pulse in $d N(\tau)$ is proportional to $d \tau$, but for more than one pulse in $d N(\tau)$ is of a negligible smaller order of magnitude in $d \tau$. Hence moments of temperature distribution and its thermal stresses can be obtained as

$$
\begin{aligned}
\varepsilon\left\{\sigma_{r r}\right\}= & \sum_{m=1}^{\infty} \sum_{n=1}^{\infty} 2 G B_{n} \wp_{m n}\left\langle\left[-\alpha_{n} J_{1}\left(\alpha_{n} r\right) / r-\gamma_{m}^{2} J_{0}\left(\alpha_{n} r\right)\right]\right. \\
& \times \sinh \left[\gamma_{m}(z+\ell / 2)\right]+\gamma_{m}\left\{K _ { m 1 } \operatorname { c o s h } \left[\gamma_{m}(z+\ell / 2)\right.\right. \\
& \left.\left.+K_{m} 2 \sinh \left[\gamma_{m}(z+\ell / 2)\right]\right\}\right\rangle \\
& \left.\times v\left[\alpha_{n} J_{1}\left(\alpha_{n} r\right) / r+\gamma_{m}^{2} J_{0}\left(\alpha_{n} r\right)\right]\right\rangle \\
\varepsilon\left\{\sigma_{\theta \theta}\right\}= & \sum_{m=1}^{\infty} \sum_{n=1}^{\infty} 2 G B_{n} \wp_{m n}\left\langle\gamma_{m}^{2} J_{0}\left(\alpha_{n} r\right) \sinh \left[\gamma_{m}(z+\ell / 2)\right]\right. \\
& +\gamma_{m}\left[(v-1) \alpha_{n} J_{1}\left(\alpha_{n} r\right) / r+v \gamma_{m}^{2} J_{0}\left(\alpha_{n} r\right)\right] \\
& \times\left\{K_{m 1} \cosh \left[\gamma_{m}(z+\ell / 2)+K_{m 2} \sinh \left[\gamma_{m}(z+\ell / 2)\right]\right\}\right\rangle \\
\varepsilon\left\{\sigma_{z z}\right\}= & \sum_{m=1}^{\infty} \sum_{n=1}^{\infty} 2 G B_{n} \wp_{m n}\left\langle-\alpha_{n} J_{1}\left(\alpha_{n} r\right) \sinh \left[\gamma_{m}(z+\ell / 2)\right] / r\right. \\
+ & \gamma_{m}\left[(2-v) \alpha_{n} J_{1}\left(\alpha_{n} r\right) / r+(1-v) \gamma_{m}^{2} J_{0}\left(\alpha_{n} r\right)\right] \\
& \times\left\{K_{m 1} \cosh \left[\gamma_{m}(z+\ell / 2)+K_{m 2} \sinh \left[\gamma_{m}(z+\ell / 2)\right]\right\}\right\rangle \\
& \infty \sigma_{r z}^{\infty}=\sum_{m=1}^{\infty} 2 G B_{n} \alpha_{n} \gamma_{m} \wp_{m n} J_{1}\left(\alpha_{n} r\right) \\
& \times\left\langle\operatorname { c o s h } \left[\gamma_{m}(z+\ell / 2)-v \gamma_{m}\left\{K _ { m 1 } \operatorname { s i n h } \left[\gamma_{m}(z+\ell / 2)\right.\right.\right.\right. \\
& \left.\left.+K_{m 2} \cosh \left[\gamma_{m}(z+\ell / 2)\right]\right\}\right\rangle
\end{aligned}
$$

where $\wp_{m n}=[1-\exp (-\mathrm{t} \omega)]\{\theta+I\} / \omega$

\subsection{Correlation Subjected to Random Pulses}

Similarly, in the case of product density of degree two, we assume

$$
\begin{aligned}
& \varepsilon\left\{d N\left(\tau_{1}\right) d N\left(\tau_{2}\right)\right\}=\bar{C}^{2}, \\
& \varepsilon\left\{\psi\left(\tau_{1}\right) \psi\left(\tau_{2}\right)\right\}=\exp \left\{-\mu\left(\tau_{2}-\tau_{1}\right)\right\}
\end{aligned}
$$

where $\bar{C}$ is the constant average density and $\mu>0, \tau_{2}>\tau_{1}$.

Hence the correlation of temperature distribution and its thermal stresses can be obtained as

$$
\begin{aligned}
& \varepsilon\left\{\sigma_{r \eta} \sigma_{r r_{2}}\right\}=\sum_{m, m^{\prime}}^{\infty} \sum_{n, n^{\prime}}^{\infty} 4 G^{2} B_{n} \wp_{m n m^{\prime}} n^{\prime} \\
& \times\left\langle\left[-\alpha_{n} J_{1}\left(\alpha_{n} r\right) / r-\gamma_{m}^{2} J_{0}\left(\alpha_{n} r\right)\right]\right. \\
& \times \sinh \left[\gamma_{m}(z+\ell / 2)\right]+\gamma_{m}\left\{K_{m 1}\right. \\
& \times \cosh \left[\gamma_{m}(z+\ell / 2)+K_{m 2}\right. \\
& \left.\times \sinh \left[\gamma_{m}(z+\ell / 2)\right]\right\} v\left[\alpha_{n} J_{1}\left(\alpha_{n} r\right) / r\right. \\
& \left.+\gamma_{m}^{2} J_{0}\left(\alpha_{n} r\right)\right]\left[-\alpha_{n} J_{1}\left(\alpha_{n^{\prime}} r\right) / r-\gamma_{m}^{2}\right. \text {, } \\
& \left.\times J_{0}\left(\alpha_{n^{\prime}} r\right)\right] \sinh \left[\gamma_{m^{\prime}}(z+\ell / 2)\right]+\gamma_{m^{\prime}} \\
& \times\left\{K _ { m 1 } \operatorname { c o s h } \left[\gamma_{m}(z+\ell / 2)+K_{m 2}\right.\right. \\
& \left.\times \sinh \left[\gamma_{m}(z+\ell / 2)\right]\right\} \\
& \left.\times v\left[\alpha_{n^{\prime}} J_{1}\left(\alpha_{n^{\prime}} r\right) / r+\gamma_{m^{\prime}}^{2} J_{0}\left(\alpha_{n^{\prime} r}\right)\right]\right\rangle \\
& \varepsilon\left\{\sigma_{\theta \theta_{1}} \sigma_{\theta \theta_{2}}\right\}=\sum_{m=1}^{\infty} \sum_{n=1}^{\infty} 4 G^{2} B_{n} \wp_{m n m^{\prime}} n^{\prime} \\
& \times\left\langle\left\{\gamma_{m}^{2} J_{0}\left(\alpha_{n} r\right) \sinh \left[\gamma_{m}(z+\ell / 2)\right]\right.\right. \\
& +\gamma_{m}\left[(v-1) \alpha_{n} J_{1}\left(\alpha_{n} r\right) / r+v \gamma_{m}^{2} J_{0}\left(\alpha_{n} r\right)\right\} \\
& \times\left\{K_{m 1} \cosh \left[\gamma_{m}(z+\ell / 2)\right]+K_{m 2}\right. \\
& \left.\times \sinh \left[\gamma_{m}(z+\ell / 2)\right]\right\}\left\{\gamma_{m^{\prime}}^{2} J_{0}\left(\alpha_{n^{\prime} r}\right)\right. \\
& \times \sinh \left[\gamma_{m^{\prime}}(z+\ell / 2)\right]+\gamma_{m^{\prime}}\left\{(v-1) r^{-1}\right. \\
& \left.\times \alpha_{n^{\prime}} J_{1}\left(\alpha_{n^{\prime} r}\right)+v \gamma_{m^{\prime}}^{2} J_{0}\left(\alpha_{n^{\prime} r}\right)\right\} \\
& \times\left\{K _ { m 1 } \operatorname { c o s h } \left[\gamma_{m^{\prime}}(z+\ell / 2)\right.\right. \\
& \left.\left.+K_{m 2} \sinh \left[\gamma_{m^{\prime}}(z+\ell / 2)\right]\right\}\right\rangle \\
& \varepsilon\left\{\sigma_{z z_{1}} \sigma_{z z 2}\right\}=\sum_{m=1}^{\infty} \sum_{n=1}^{\infty} 4 G^{2} B_{n} \wp_{m n m^{\prime}} n^{\prime} \\
& \times\left\langle\left[-\alpha_{n} J_{1}\left(\alpha_{n} r\right) \sinh \left[\gamma_{m}(z+\ell / 2)\right] / r\right.\right. \\
& +\gamma_{m}\left\{(2-v) \alpha_{n} J_{1}\left(\alpha_{n} r\right) / r+(1-v) \gamma_{m}^{2}\right. \\
& \left.\times J_{0}\left(\alpha_{n} r\right)\right\}\left\{K _ { m 1 } \operatorname { c o s h } \left[\gamma_{m}(z+\ell / 2)\right.\right. \\
& \left.\left.+K_{m 2} \sinh \left[\gamma_{m}(z+\ell / 2)\right]\right\}\right] \\
& \times\left[-\alpha_{n^{\prime}} J_{1}\left(\alpha_{n^{\prime}} r\right) \sinh \left[\gamma_{m^{\prime}}(z+\ell / 2)\right] / r\right. \\
& +\gamma_{m^{\prime}}\left\{(2-v) \alpha_{n^{\prime}} J_{1}\left(\alpha_{n^{\prime} r}\right) / r+(1-v)\right. \\
& \left.\times \gamma_{m}^{2} J_{0}\left(\alpha_{n} r\right)\right\}\left\{K _ { m 1 } \operatorname { c o s h } \left[\gamma_{m}{ }^{\prime}(z+\ell / 2)\right.\right. \\
& \left.+K_{m 2} \sinh \left[\gamma_{m^{\prime}}(z+\ell / 2)\right]\right\rangle
\end{aligned}
$$




$$
\begin{aligned}
& \varepsilon\left\{\sigma_{r z_{1}} \sigma_{r z_{2}}\right\}=\sum_{m=1}^{\infty} \sum_{n=1}^{\infty} 4 G^{2} B_{n} \alpha_{n} \alpha_{n^{\prime}} \gamma_{m} \gamma_{m^{\prime}} \\
& \times \wp_{m n m^{\prime} n^{\prime} J_{1}\left(\alpha_{n} r\right) J_{1}\left(\alpha_{n} r\right)} \\
& \times\left\langle\cosh \left[\gamma_{m}(z+\ell / 2)\right]-v \gamma_{m}\left\{K_{m 1}\right.\right. \\
& \times \sinh \left[\gamma_{m}(z+\ell / 2)+K_{m 2}\right. \\
& \left.\times \cosh \left[\gamma_{m}(z+\ell / 2)\right]\right\}\left\{\cosh \left[\gamma_{m}(z+\ell / 2)\right]\right. \\
& -v \gamma_{m^{\prime}}\left\{K _ { m 1 } \operatorname { s i n h } \left[\gamma_{m}(z+\ell / 2)\right.\right. \\
& \left.\left.+K_{m 2} \cosh \left[\gamma_{m^{\prime}}(z+\ell / 2)\right]\right\}\right\rangle
\end{aligned}
$$

where$$
\wp_{m n m^{\prime} n^{\prime}}=\left\langle\frac{e^{-\omega t_{1}-t_{2} \omega^{\prime}}\left(e^{\omega t_{2}}-e^{\omega \tau_{1}}\right)\left(-1+e^{t_{1} \omega^{\prime}}\right) \theta^{2}}{\omega \omega^{\prime}}\right.
$$$$
+\frac{e^{-(\mu+\omega) t_{1}-\omega^{\prime} t_{2}}}{\left[\beta^{2}+(\mu+\omega)^{2}\right]\left(\mu-\omega^{\prime}\right)}\left(e^{\mu t_{1}}-e^{\omega^{\prime} t_{1}}\right)
$$$$
\times\left\{-e^{(\mu+\omega) t_{2}} \beta \cos \left(\beta t_{2}\right)+e^{(\mu+\omega) \tau_{1}} \beta \cos \left(\beta \tau_{1}\right)\right.
$$$$
\left.+(\mu+\omega)\left[e^{(\mu+\omega) t_{2}} \sin \left(\beta t_{2}\right)-e^{(\mu+\omega) \tau_{1}} \sin \left(\beta \tau_{1}\right)\right]\right\}
$$$$
+\frac{e^{-\omega t_{1}-t_{2} \omega^{\prime}} \theta^{2}\left[e^{\omega t_{1}}\left(-1+e^{t_{1} \omega^{\prime}}\right) \omega-\left(-1+e^{\omega t_{1}}\right) \omega^{\prime}\right]}{\omega \omega^{\prime}\left(\omega+\omega^{\prime}\right)}
$$$$
+\frac{1}{\beta^{2}+(\mu+\omega)^{2}}\left\{-e^{-\omega t_{1}-\omega^{\prime} t_{2}}\right.
$$$$
\times\left[\frac{e^{(\mu+\omega) t_{1}}\left[\beta \cos \left(\beta t_{1}\right)-(\mu+\omega) \sin \left(\beta t_{1}\right)\right]}{\mu-\omega^{\prime}}\right.
$$$$
\left.+\frac{\beta\left(\mu+2 \omega+\omega^{\prime}\right)}{\beta^{2}+\omega^{2}+2 \omega \omega^{\prime}+\omega^{\prime 2}}\right]+e^{-\mu t_{1}-\omega t_{1}+\omega^{\prime}\left(t_{1}-t_{2}\right)}
$$$$
\times\left[\frac{e^{(\mu+\omega) t_{1}}\left[\beta \cos \left(\beta t_{1}\right)-(\mu+\omega) \sin \left(\beta t_{1}\right)\right]}{\mu-\omega^{\prime}}\right.
$$$$
+\frac{e^{(\mu+\omega) t_{1}} \beta \cos \left(\beta t_{1}\right)\left(\mu+2 \omega+\omega^{\prime}\right)}{\beta^{2}+\omega^{2}+2 \omega \omega^{\prime}+\omega^{\prime 2}}
$$$$
\left.\left.-\frac{e^{(\mu+\omega) t_{1}} \sin \left(\beta t_{1}\right)\left[-\beta^{2}+\omega(\mu+\omega)+(\mu+\omega) \omega^{\prime}\right]}{\beta^{2}+\omega^{2}+2 \omega \omega^{\prime}+\omega^{\prime 2}}\right]\right\}
$$$$
\left.+\frac{e^{-\omega t_{1}-t_{2} \omega^{\prime}}\left[-1+e^{t_{1}\left(\omega+\omega^{\prime}\right)}\right]}{\omega+\omega^{\prime}}\right\rangle
$$

\section{Numerical Results and Discussion}

Introducing non-dimensional variables as

$$
\begin{aligned}
& \Theta=T / T_{R}, \zeta=[z-(-\ell / 2)] / a, \\
& \rho=r / a, \bar{\tau}=\kappa t / a^{2}, \\
& \left(\bar{\sigma}_{r r}, \bar{\sigma}_{\theta \theta}, \bar{\sigma}_{z z}, \bar{\sigma}_{r z}\right)=\left(\sigma_{r r}, \sigma_{\theta \theta}, \sigma_{z z}, \sigma_{r z}\right) / E \alpha_{t} T_{R}
\end{aligned}
$$

with parameters $a=1 \mathrm{~m}, \ell=0.8 \mathrm{~m}$ and the reference temperature is $T_{R}=32^{\circ} \mathrm{C}$. The thermo-mechanical properties are considered as modulus of elasticity $E=70$ $\mathrm{GPa}$, Poisson's ratio $v=0.35$, thermal expansion coefficient $\alpha_{T}=23 \times 10-6 / 0 \mathrm{C}$, thermal diffusivity $\kappa=$ $84.18 \times 10-6 \mathrm{~m} 2 \mathrm{~s}-1$ and thermal conductivity $\lambda=204.2$ Wm-1K-1, Shear modulus $G=25.5 \mathrm{GPa}, t_{1}=0.5 \mathrm{sec}$, $t_{2}=0.9 \mathrm{sec}, \rho_{0}=0.6, \mu=1(>0)$.
Here $3.831,7.015,10.173,13.323,16.470,19.615,22.76$, 25.903, 29.047, 32.189, 35.332, 38.474, 41.617, 44.789, 47.905 are the real and positive roots of the transcendental equation $J_{1}\left(\alpha_{n} a\right)=0$. In order to portray the temperature solution to be meaningful, the series expressed in Eq. (1), ought to converge for all $0 \leq r \leq a$ and $-\ell / 2 \leq z \leq \ell / 2$. However, it should be noted that the Bessel series $\sum A_{n} J_{0}\left(\alpha_{n} r\right)$ of $f(r)$ behaves precisely like the Fourier series of $f(r)$, at any point to $0 \leq r \leq a$, provided $A_{n} J_{0}\left(\alpha_{n} r\right) \rightarrow 0(r \rightarrow \infty)$, and also $J_{0}\left(\alpha_{n} r\right)$ is bounded function of $r$, where the numbers $\alpha_{1}, \alpha_{2}, \ldots \alpha_{n}, \ldots$ form a monotone increasing succession tending to infinity, and are determined as the positive roots of the transcendental equation $J_{1}\left(\alpha_{n} a\right)=0$. By using the Maclaurin series for $f(z)$ and Ratio test, it is observed that the radius of convergence is less than 1 (regardless of the value of $z$ ). Thus from above, it is clear that $A_{n} J_{0}\left(\alpha_{n} r\right) \rightarrow 0$ and $\sinh \left(\gamma_{m} z\right) \rightarrow 0$ as $n \rightarrow \infty$ and $m \rightarrow \infty$, which shows that $T(r, z, t)$ also converges to the finite value as $n \rightarrow \infty$ and $m \rightarrow \infty$. Numerically it had been determined that the series solutions converge provided if we have a tendency to take an adequate range of terms within the series. Irrespective of the thickness of the plate, the series solution is given here will be definitely convergent. To summarize, our convergence statement says that series solutions collapse onto the exact solution with the error tending to zero at all indices if we tend to take an adequate range of terms. In other words, the convergence rate is faster if we shrink the step smaller and smaller. Thus, for computational simplicity, we have considered 15 eigenvalues, and the numerical results were obtained for the figures with the help of MATHEMATICA software. Figure 2 shows the correlation of temperature distribution in the thick plate plotted against the dimensionless axial coordinate (i.e. $\zeta$-direction) for different values of the dimensionless radius $\rho=0.2,0.4,0.6,0.8,1$. This figure indicates that in the initial state the correlation of temperature is zero then gradually increases up to the central part and start decreasing to the other part till it reaches $\zeta=0.6$ as in agreement with Ahmadi [11], due to the randomness of temperature. The temperature in the lower curved surface again starts gradually increasing due to the external random sectional heat supply at $\zeta=0.8$.

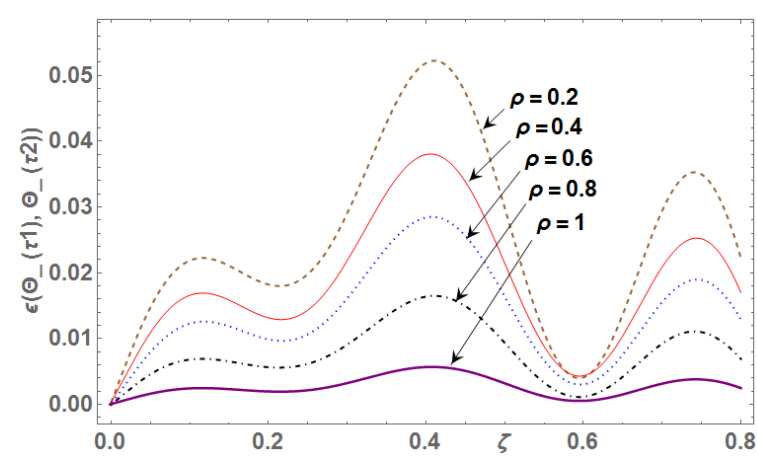

Fig. 2 Correlation of dimensionless temperature distribution in the axial direction. 


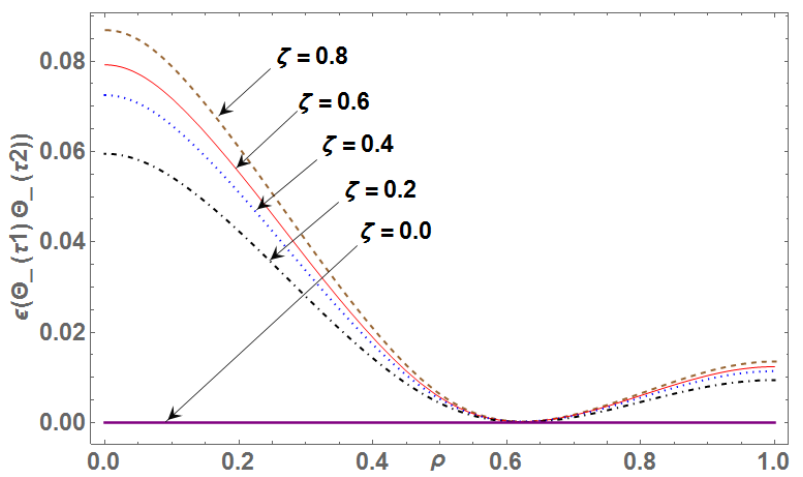

Fig. 3 Correlation of dimensionless temperature distribution in the radial direction.

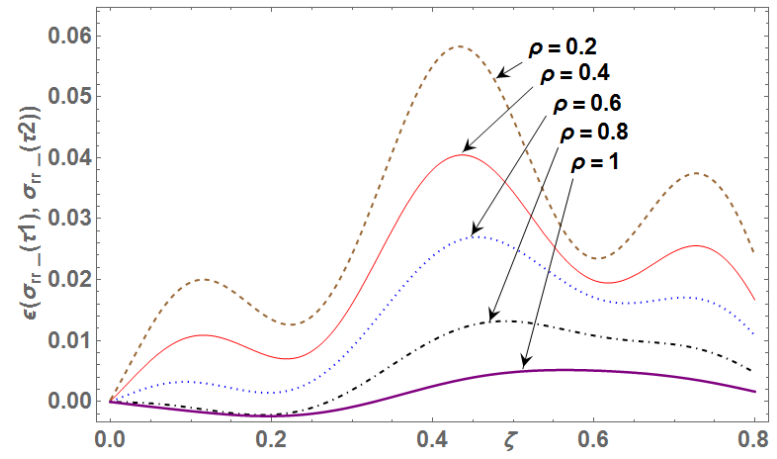

Fig. 4 Correlation of dimensionless radial stress in the axial direction.

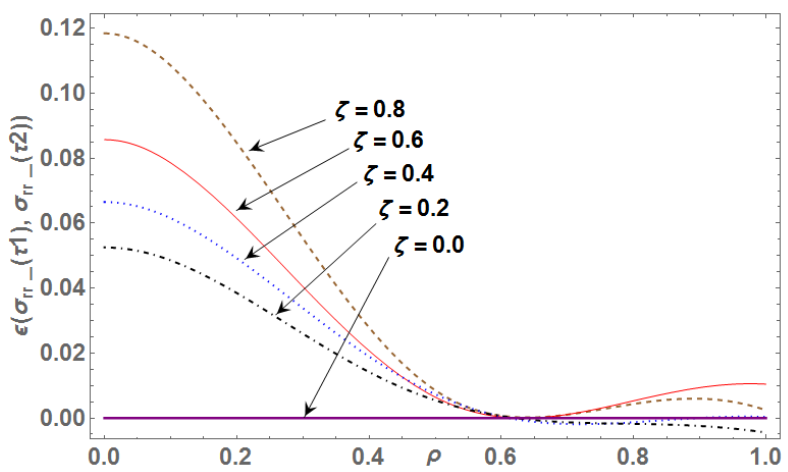

Fig. 5 Correlation of dimensionless radial stress in the radial direction.

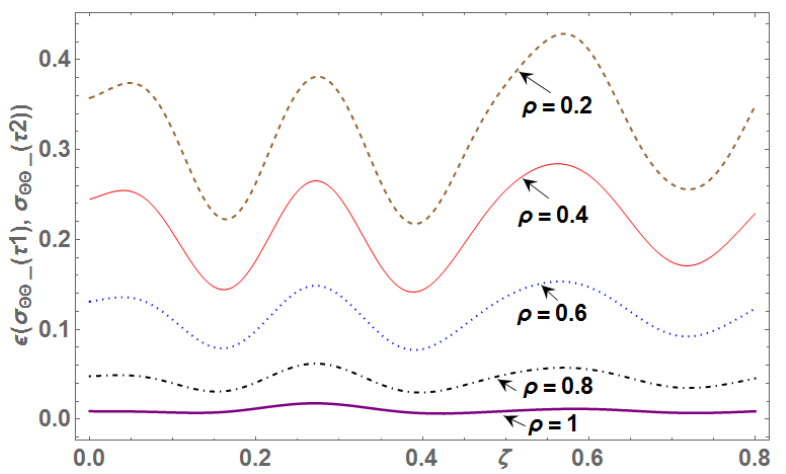

Fig. 6 Correlation of the dimensionless tangential stress in the axial direction.

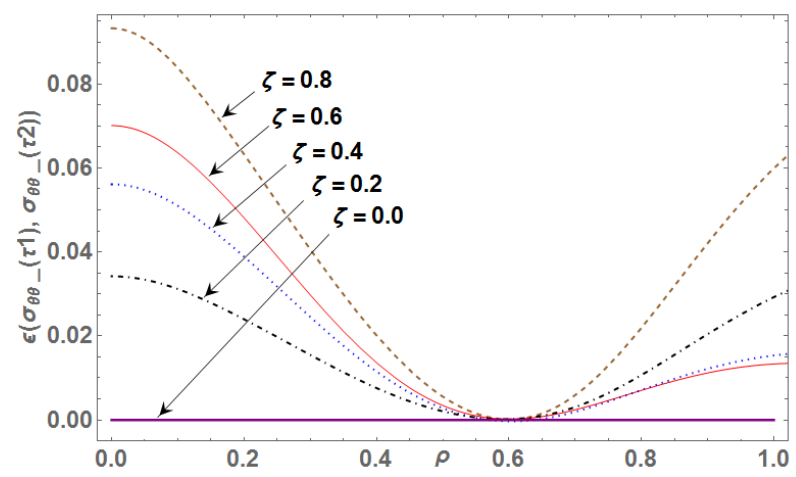

Fig. 7 Correlation of dimensionless tangential stress in the radial direction.

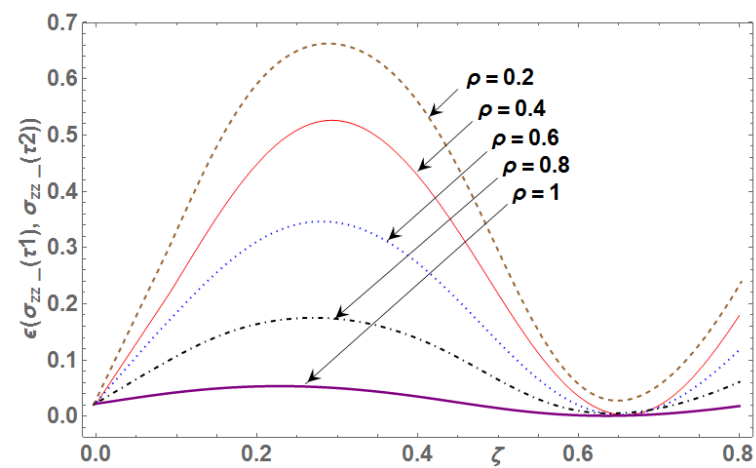

Fig. 8 Correlation of dimensionless axial stress in the axial direction.

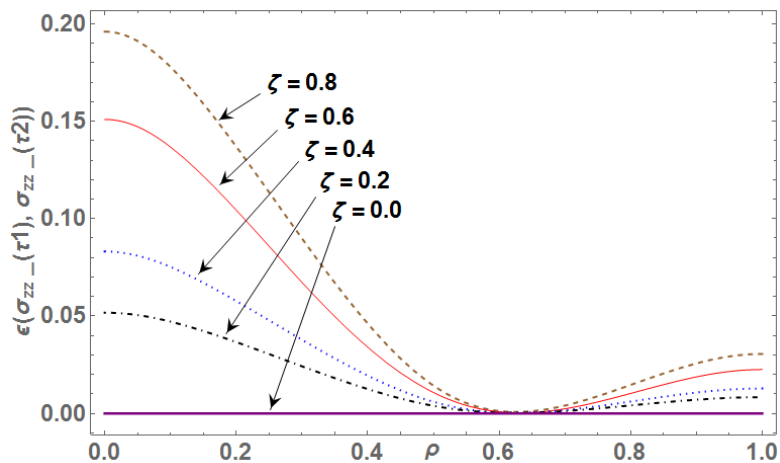

Fig. 9 Correlation of dimensionless axial stress in the radial direction.

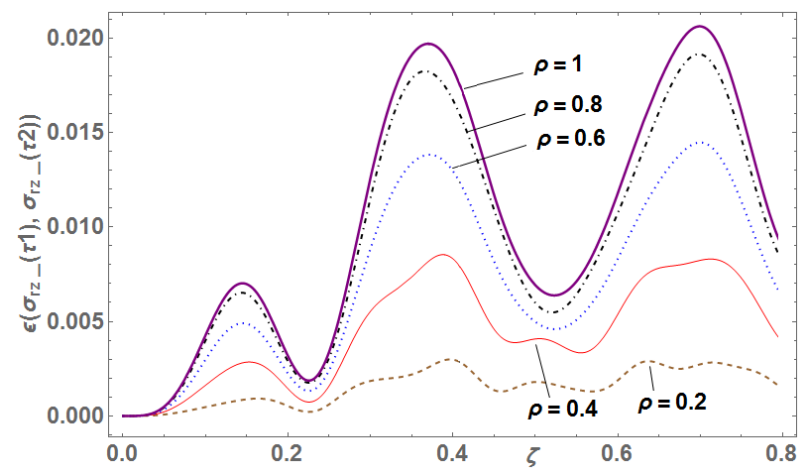

Fig. 10 Correlation of the dimensionless shear stress in the axial direction. 


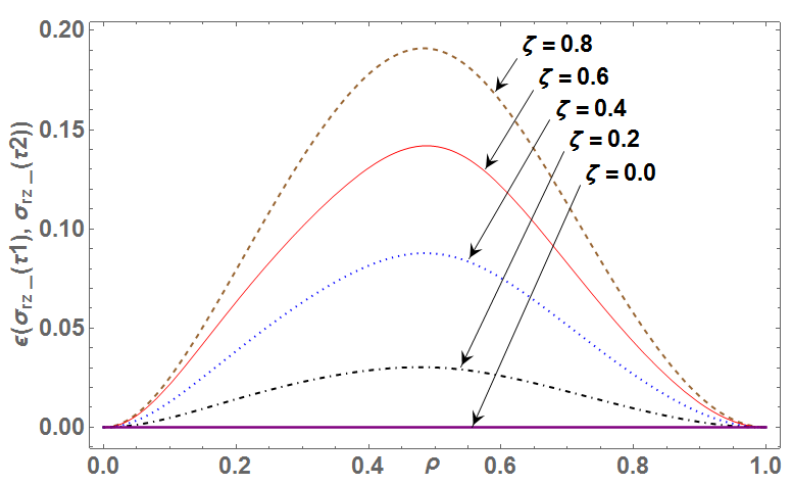

Fig. 11 Correlation of the dimensionless shear stress in the radial direction.

Figure 3 shows the correlation of temperature distribution in the dimensionless radial coordinate $(\rho$-direction) for five different locations of dimensionless thickness $\zeta=0,0.2,0.4,0.6,0.8$. It is observed from the figure that due to random surface temperature distribution on the upper surface; it is observed that the correlation of temperature is high in the central region $\left(0<\rho<\rho_{0}\right)$ of the plate, as expected. Moreover, a small temperature gradient appears towards the outer insulated radius due to the accumulation of thermal energy dissipated by the internal heat source and the sectional heat supply. The influence of dimensionless temperature is well observed in figure 4 which displays the high correlation of radial stresses along the axial direction, and moderate correlation can be seen around the unheated region. Figure 5 shows radial stresses along the radial direction that was found to be maximum due to the tensile stress on the curved surface at $\rho=0$ and the compressive stress occurring at the curved part at $\rho=1$ of the thick plate and its absolute value increases at different location of the axial direction. The effects of random temperature were also well observed in figure 6 , which illustrates the distribution of the correlation of tangential stress along the axial direction for different values of $\rho$. It can be seen that the nature of the graph is sinusoidal that could be owing due to thermal expansion. Figure 7 displays the distribution of tangential stress along the radial direction at various location of thickness, and very well versed similarity was found with the radial stress in the radial direction as shown in figure 5. Figure 8 thoroughly demonstrates the correlation of the axial stress in the axial direction for different values of $\rho$. It is seen that the axial stress attains the maximum expansion then gradually decreases up to $\zeta=0.7$ at different locations $\rho=0.2,0.4,0.6,0.8,1$, then gradually increases until it reaches $\zeta=0.8$ along the $\zeta-$ direction. Figure 9 illustrates that the absolute value of axial stresses decreases towards the mid part of thick plate with the different axial points, but increase at the outer edge. Similar radial inherent nature was also found in figures 3,5 and 7 along the radial direction for various value of $\zeta$. Figure 10 expresses the correlation of shear stress at different location of $\rho$ and it was found that the nature of the distribution is sinusoidal and increases monotonically along the axial direction. These changes could owe due to thermal expansion. Fig. 11 shows the correlation of shear stress in a radial direction for different values of $\zeta$. It is observed that the nature of the graph is bell-shaped. The correlation is initially zero at $\rho=0$ and it slowly increases from the centre of the plate and is attaining peak value in the region $\rho=0.5$, then slows down to zero at $\rho=1$. It might occur due to the accumulation of heat energy dissipated by sectional and internal heat supply which further decreases at the two extreme ends.

\section{Conclusion}

In the present work, we have investigated thermal stresses in a thick circular plate subjected to the random temperature distribution. Moments and correlation of temperature distribution and thermal stresses are obtained using Poisson's distribution. Generally, due to the random nature of temperature, the accurate prediction of thermal or mechanical loads on structural components is difficult. Hence, we have adopted the technique of moments and correlation for thermoelastic analysis. During the investigation, all the critical factors are taken in experimental favour. It is clearly observed that the nature of the figures of temperature and all stresses are sinusoidal when plotted for axial direction and exponentially decayed when plotted for radial direction except for shear stress, which is observed to be a bell-shaped curve.

The under given future scope can be taken for pursuing further studies

- This study can further be applied to multiple time intervals using multivariate correlation analysis.

- Particular cases of special interest can be studied by taking the material properties in the equations of temperature and thermal stresses to be nonhomogeneous as well as by keeping the size of the time interval low and high for numerical analysis.

- This type of theoretical analysis may be used for the high cycle temperature fluctuations in reactors, gas turbines and fluctuating voltages in thermionic valves.

\section{Acknowledgements:}

The authors are grateful to the reviewer and editor for their valuable suggestion and constructive comments which resulted in revising the paper to its present form.

\section{Nomenclature}

$$
\begin{array}{ll}
(r, \theta, z) & =\text { Cylindrical coordinates } \\
(x, y, z) & =\text { Cartesian coordinates } \\
r & =\sqrt{x^{2}+y^{2}} \\
\theta & =\tan ^{-1}(y / x) \\
k & =\text { Thermal conductivity. }
\end{array}
$$

$T(r, z, t)=$ Temperature distribution at any time.

$(\lambda, \mu)=$ Lame's constant

$J_{0} \quad=$ First kind Bessel functions with order zero

$J_{1} \quad=$ First kind Bessel functions with order one

$Y_{0} \quad=$ Second kind Bessel functions with order zero

\section{References:}

[1] R. Chiba (2012), "Stochastic analysis of heat conduction and thermal stresses in solids: A Review," Heat Transfer Phenomena and Applications, S.N. Kazi (ed), IntechOpen, pp. 243-266. https://doi.org/10.5772/50994

[2] P.M. Mathews, S.K. Srinivasan (1956), “Ordinary linear differential equations involving random 
functions," Proc. Ind. Acad. Sci., vol. 43, pp. 4-20. https://doi.org/10.1007/BF03052627

[3] H. Parkus (1962), "Wärmespannungen bei zufallsabhängiger Oberflächentemperatur," ZAMM, vol. 42, pp. 499-507. https://doi.org/10.1002/zamm.19620421008

[4] J.C. Samuels (1966), "Heat conduction in solids with random external temperatures and/or random internal heat generation," Int J Heat Mass Tran., vol. 9, pp. 301-314. 9310(66)90076-7 https://doi.org/10.1016/0017-

[5] S.K. Srinivasan, R. Subramanian, S. Kumaraswamy (1969), "Unsteady motion of a viscous fluid subjected to random pulses," Arch. Mech. Stos., vol. 21, pp. 191-198.

[6] H.M. Hung (1969), "Heat transfer of thin fins with stochastic root temperature," J Heat Tran.. , vol. 91, pp. 129-134. https://doi.org/10.1115/1.3580068

[7] R.A. Heller (1976), "Temperature response of an infinitely thick slab to random surface temperatures," Mech Res Comm., vol. 3, pp. 379-385. https://doi.org/10.1016/0093-6413(76)90097-5

[8] M.P. Lenyuk, M.I. Bukatar, L.K. Shelyag (1976), "Random thermal stresses in an elastic halfspace," Int Appl Mech., vol. 12, pp. 452-458. https://doi.org/10.1007/BF00882450

[9] M.P. Lenyuk, L.K. Shelyag (1978), "Stress state of symmetric elastic body under random thermal perturbations," Int Appl Mech., vol. 14, pp. 1137 1142.

[10] G.A. Becus (1977), "Random generalized solutions to the heat equation," J. Math. Analysis Appl., vol. 60, pp. 93-102. https://doi.org/10.1016/0022247X(77)90051-8

[11] G. Vasseur, R.N. Singh (1986), "Effects of random horizontal variations in radiogenic heat source distribution on its relationship with heat flow," $J$ Geophys Res., vol. 91, pp. 10397-10404. https://doi.org/10.1029/JB091iB10p10397

[12] S.B. Nielsen (1987), "Steady state heat flow in a random medium and the linear heat flow heat production relationship," Geophys Res Letters, vol. 14, $\quad$ pp. 318-21.
[13] V.I. Val'kovskaya, M.P. Lenyuk (1996), "Stochastic nonstationary temperature fields in a solid circularcylindrical two-layer plate," J Math Sci., vol. 79, pp. 1483-1487. https://doi.org/10.1007/BF02362811

[14] Y. Sugano, T. Kanno, R. Chiba (2002), "Stochastic thermal deformation and thermal stress in a laminated plate including FGM layer under random surface temperatures," Trans Jpn Soc Mech Eng., vol. 68A, pp. 1588-1593. https://doi.org/10.1299/kikaia.68.1588

[15] Y. Sugano, R. Chiba (2002), “A stochastic analysis of temperature and thermal stress in functionally graded plates with randomly varying initial temperature," $J$ Soc Mater Sci., vol. 51, pp. 653-658. https://doi.org/10.2472/jsms.51.653

[16] Y. Sugano, R. Chiba (2007), "Stochastic thermoelastic problem of a functionally graded plate under random temperature load," Arch Appl Mech., vol. 77, pp. 215-227. https://doi.org/10.1007/s00419006-0088-7

[17] Y. Sugano, R. Chiba (2010), "Thermal stresses in bodies with random initial temperature distribution," Math. Mech. Solids, vol. 15, pp. 258-276. https://doi.org/10.1177/1081286508100497

[18] Y. Sugano, R. Chiba (2014), "Encyclopedia of thermal stresses. In: Hetnarski RB (ed) Stochastic analysis of thermal stresses in bodies. Springer, Dordrecht," pp 4564-4573. https://doi.org/10.1007/978-94-007-2739-7_237

[19] R. Chiba (2007), "Stochastic thermal stresses in an annular disc with spatially random heat transfer coefficients on upper and lower surfaces," Acta Mechanica, vol. 194, pp. 67-82. https://doi.org/10.1007/s00707-007-0461-9

[20] N. Noda, Y. Takeuti, K. Takakura (1982), “Transient thermal stress problem in a thick circular plate due to sectioned heat supply on its flat surface," Transactions of the Japan Society of Mechanical Engineers, vol. 48, pp. 329-338.

[21] G. Ahmadi (1974), "Heat Conduction in Solids with Random Initial Conditions," J. Heat Transf. Trans. ASME, vol. 96, pp. 474-477. https://doi.org/10.1115/1.3450230 https://doi.org/10.1029/GL014i003p00318 